TITLE:

\title{
Direct evidence for the two-phonon bound states on the $\mathrm{H} / \mathrm{Ni}(111)$ surface
}

\section{$\operatorname{AUTHOR}(S)$ :}

Okuyama, H; Ueda, T; Aruga, T; Nishijima, M

\section{CITATION:}

Okuyama, $\mathrm{H}$...[et al]. Direct evidence for the two-phonon bound states on the H/Ni(111) surface. PHYSICAL REVIEW B 2001, 63(23): 233404.

\section{ISSUE DATE:}

2001-06-15

URL:

http://hdl.handle.net/2433/49835

\section{RIGHT:}

Copyright 2001 American Physical Society 
PHYSICAL REVIEW B, VOLUME 63, 233404

\title{
Direct evidence for the two-phonon bound states on the H/Ni(111) surface
}

\author{
H. Okuyama, T. Ueda, T. Aruga, and M. Nishijima* \\ Department of Chemistry, Graduate School of Science, Kyoto University, Kyoto 606-8502, Japan \\ (Received 6 October 2000; revised manuscript received 16 January 2001; published 14 May 2001)
}

\begin{abstract}
We have investigated multiple losses and overtones of $\mathrm{H}$ vibrations at $\mathrm{Ni}(111)$ by using electron-energy-loss spectroscopy. We mapped the energies along the $\bar{\Gamma} \bar{M}$ direction of the surface Brillouin zone, and found two branches outside the two-phonon continua. This gives direct evidence of the existence of two-phonon bound states. Applying a one-dimensional model of coupled anharmonic oscillators, we derive the anharmonicity and discuss the localized property of the bound states.
\end{abstract}

DOI: 10.1103/PhysRevB.63.233404

PACS number(s): 68.35.Ja, 68.43.Pq

\section{INTRODUCTION}

The overtone spectra for $\mathrm{H}$ vibrations at surfaces provide information on the vibrational anharmonicities, which are indispensable for a knowledge of the potential shapes, and thereby for an understanding of the dynamical properties of $\mathrm{H}$ on surfaces. For isolated species including gas-phase molecules, the vibrational anharmonicities can be directly evaluated from the observed overtone energies, where the extraction of physical parameters such as the dissociation energies are straightforward through the use of the Birge-Sponer extrapolation. ${ }^{1}$ On the other hand, adsorbed $\mathrm{H}$ in the monolayer regime undergoes dynamic interactions between $\mathrm{H}$ adatoms; thus we must describe the $\mathrm{H}$ vibrational states with phonon coordinates. In this scheme, the overtone is interpreted as the two-phonon state, and, when the anharmonicity is strong enough, the overtone state could be split off from the two-phonon continuum, to form a two-phonon bound state with the two quanta localized over only a few neighboring $\mathrm{H}$ adatoms. Such a localization of the vibrational quanta is closely related to, e.g., $\mathrm{H}$ desorption dynamics induced by multiphonon excitations using the scanning tunneling microscope (STM). ${ }^{2}$

The two-phonon bound states for $\mathrm{H}$ vibrations were observed in a very few adsorbate systems, e.g., $\mathrm{H} / \mathrm{Rh}(100)$ (Ref. 3) and H/Si(111). ${ }^{4}$ In both cases, however, the overtone spectra were recorded only near the $\bar{\Gamma}$ point of the surface Brillouin zone, which precluded a detailed analyses of the two-phonon bound states. As for other adsorbate systems, the overtone of the $\mathrm{C}-\mathrm{O}$ stretch was thoroughly investigated for $\mathrm{CO} / \mathrm{Ru}(001)$ by infrared-absorption spectroscopy, where the overtone was interpreted as the two-phonon bound state. ${ }^{5}$ The infrared technique, however, cannot determine the dispersions of the phonon states which provide information on the lateral coupling of the bound states.

In the present study, we mainly investigate the doubleloss and overtone regions of $\mathrm{H}$ vibrations at $\mathrm{Ni}(111)$ by using high-resolution electron-energy-loss spectroscopy (EELS). We mapped the overtone energies along the $\bar{\Gamma} \bar{M}$ direction of the surface Brillouin zone. The overtones were observed outside the two-phonon continua, which led to an interpretation of the overtones as two-phonon bound states.

\section{EXPERIMENT}

The experiments were carried out using an ultrahighvacuum chamber described in the previous paper. ${ }^{6}$ The sur- face was exposed to $\mathrm{H}_{2}$ to saturation at $90 \mathrm{~K}$, which leads to $(1 \times 1)-\mathrm{H}$ structure formation. ${ }^{7}$ The scattering plane of electrons is aligned along [ $\overline{2} 11]$. The primary energy of electrons $E_{p}$ and the incidence angle from the surface normal $\theta_{i}$ were varied from 9.5 to $25.5 \mathrm{eV}$ and $60^{\circ}$ to $80^{\circ}$, respectively. The momentum resolution was better than $0.02 \AA^{-1}$ in the dispersion measurements. All measurements were conducted at $90 \mathrm{~K}$.

\section{RESULTS}

Figure 1 shows a typical EELS spectrum for $\mathrm{Ni}(111)(1$ $\times 1)$-H. $E_{p}=9.5 \mathrm{eV}, \theta_{i}=60^{\circ}$, and the emission angle $\theta_{e}$ $=50^{\circ}$ were used $(0.11 \bar{\Gamma} \bar{M})$. The inset shows a $\mathrm{Ni}(111)(1$ $\times 1)$-H structure and the corresponding two-dimensional Brillouin zone. The dominant peaks at 954 and $1170 \mathrm{~cm}^{-1}$ were previously assigned to the asymmetric $\left(v_{a s}\right)$ and symmetric $\left(v_{s}\right)$ stretching modes of $\mathrm{H}$ adsorbed in the threefold hollow site, respectively. ${ }^{8-10}$ These assignments will later be reconfirmed by dispersion measurements for these losses.According to the transmission-channeling experiment, almost

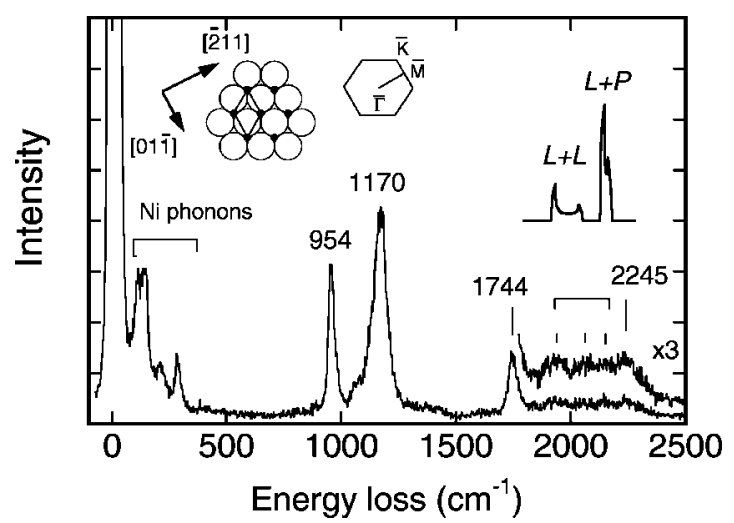

FIG. 1. Typical EELS spectra for the Ni(111)-(1×1)-H surface. $E_{p}=9.5 \mathrm{eV}, \theta_{i}=60^{\circ}$, and the emission angle $\theta_{e}=50^{\circ}$. The data are partly reproduced from Fig. 2(a) of Ref. 6. The parallel momentum transfer $\zeta\left(\equiv Q_{\|} / K_{\bar{M}}\right.$, where $\left.K_{\bar{M}}=1.457 \AA^{-1}\right)=0.11$. Another scan was made for the double-loss region with a longer data-acquisition time, and the result is shown with $\times 3$ magnification together with the calculated density of states for the free twophonon bands. The inset shows the $(1 \times 1)-\mathrm{H}$ structure and the corresponding two-dimensional Brillouin zone. 

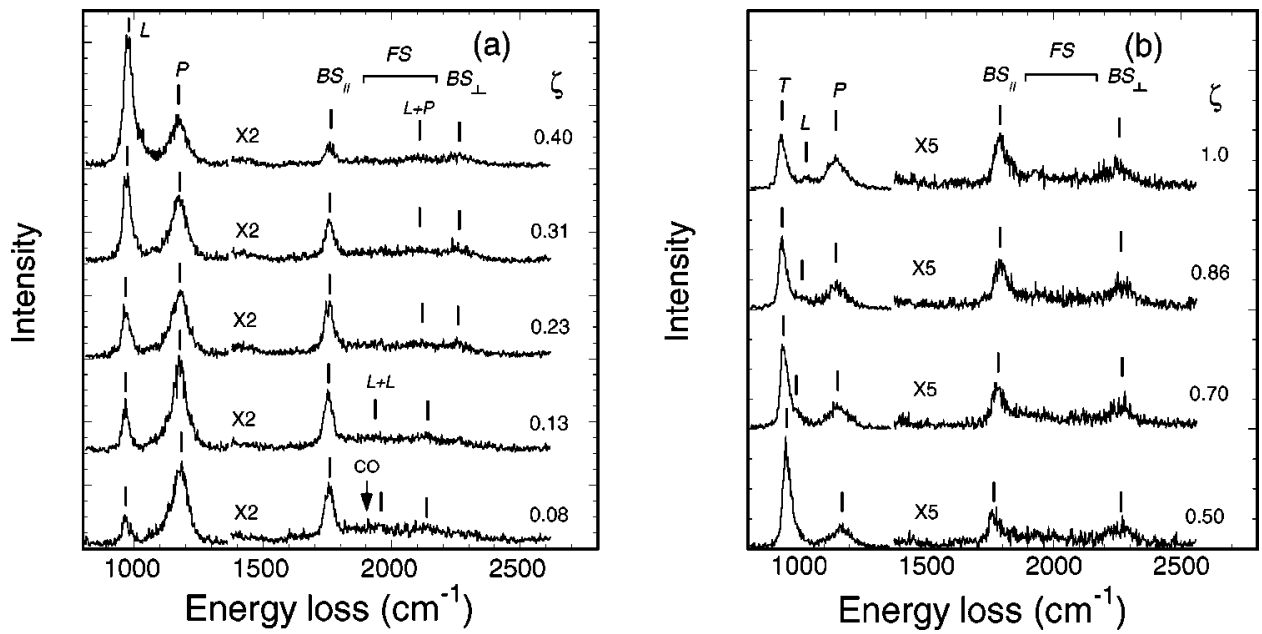

FIG. 2. EELS spectra for the $\mathrm{Ni}(111)-(1 \times 1)-\mathrm{H}$ surface as a function of $\zeta$. The primary energy and incidence angle of electrons are $9.5(25.4) \mathrm{eV}$ and $70^{\circ}\left(80^{\circ}\right)$ for (a) and (b), respectively. The notations $B S_{\|(\perp)}$ and $F S$ indicate the losses due to the two-phonon bound states and free two-phonon states, respectively. all $(90 \%)$ of the H occupies face-centered-cubic (fcc) sites. ${ }^{11}$ The shoulder observed at the lower-energy side of the $1170-\mathrm{cm}^{-1}$ loss is due to the residue of the $(2 \times 2)-2 \mathrm{H}$ phase on the surface. ${ }^{10}$ The peaks below $300 \mathrm{~cm}^{-1}$ are attributed to the Ni phonons.

In addition to the fundamental losses, we observed a sharp peak at $1744 \mathrm{~cm}^{-1}$ and a broad structure ranging from $\sim 1900$ to $2300 \mathrm{~cm}^{-1}$. It is apparent that the $1744-\mathrm{cm}^{-1}$ peak is attributed to the overtone of $v_{a s}$. The corresponding loss for D species was observed at $1310 \mathrm{~cm}^{-1}$. The broad structure is attributed to the free two-phonon band of the $\mathrm{H}$ overlayer, as will be described below.

Figure 2 shows the EELS spectra as a function of the reduced parallel momentum transfer $Q_{\|}$. The momentum transfer is represented by $\zeta \equiv Q_{\|} / K_{\bar{M}}$, where $K_{\bar{M}}$ $=1.457 \AA^{-1} . E_{p}=9.5 \mathrm{eV}, \theta_{i}=70^{\circ}$, and $E_{p}=25.5 \mathrm{eV}, \theta_{i}$ $=80^{\circ}$ were used for Figs. 2(a) and 2(b), respectively, and the emission angle was varied. The dispersion relations for the $\mathrm{H}$ vibrations are plotted in Fig. 3. First we mention the fundamental losses which correspond to the one-phonon states of $\mathrm{H}$ vibrations. $v_{\text {as }}$ exhibits an upward dispersion toward the $\bar{M}$ point, forming a longitudinal mode (denoted by $L$ ). On the other hand, the downward dispersion was observed for $v_{s}$ (denoted by $P$ ), which is due to the indirect (through metal) dynamic interaction between $\mathrm{H}$ adatoms. ${ }^{12,13}$ These dispersion behaviors reinforce the assignments of the former to the parallel-polarized mode and the latter to the perpendicularpolarized one. We observed another branch below the longitudinal mode, which is assigned as the transverse mode (denoted by $T$ ). This mode is visible only when an $E_{p}$ above $\sim 15 \mathrm{eV}$ is used, and becomes even dominant at higher $E_{p}$, as shown in Fig. 2(b). These two parallel-polarized modes ( $L$ and $T$ ) are degenerate at the $\bar{\Gamma}$ point. Though the transverse mode is forbidden under the present scattering condition (selection rule for impact scattering ${ }^{1}$ ), the finite acceptance angle in the azimuthal direction may cause the appearance of this mode at higher primary energies. The overtone of $v_{a s}$ (denoted by $B S_{\|}$) exhibits a small but significant upward dispersion. Another branch appears around $2250 \mathrm{~cm}^{-1}$ (denoted by $B S_{\perp}$ ), which is attributed to the overtone of $v_{s}$.

The data for the one-phonon states were fitted with the cosine functions, i.e., $x_{0}+x_{1} \cos (\pi \zeta)+x_{2} \cos (2 \pi \zeta)$ (solid curves). The parameters $x_{1}$ and $x_{2}$ are related to the interaction strength between the first- and second-nearest neighbors, respectively. ${ }^{14}$ Based on these curves, we calculated the free two-phonon bands as shown in Fig. 3. We obtained six bands

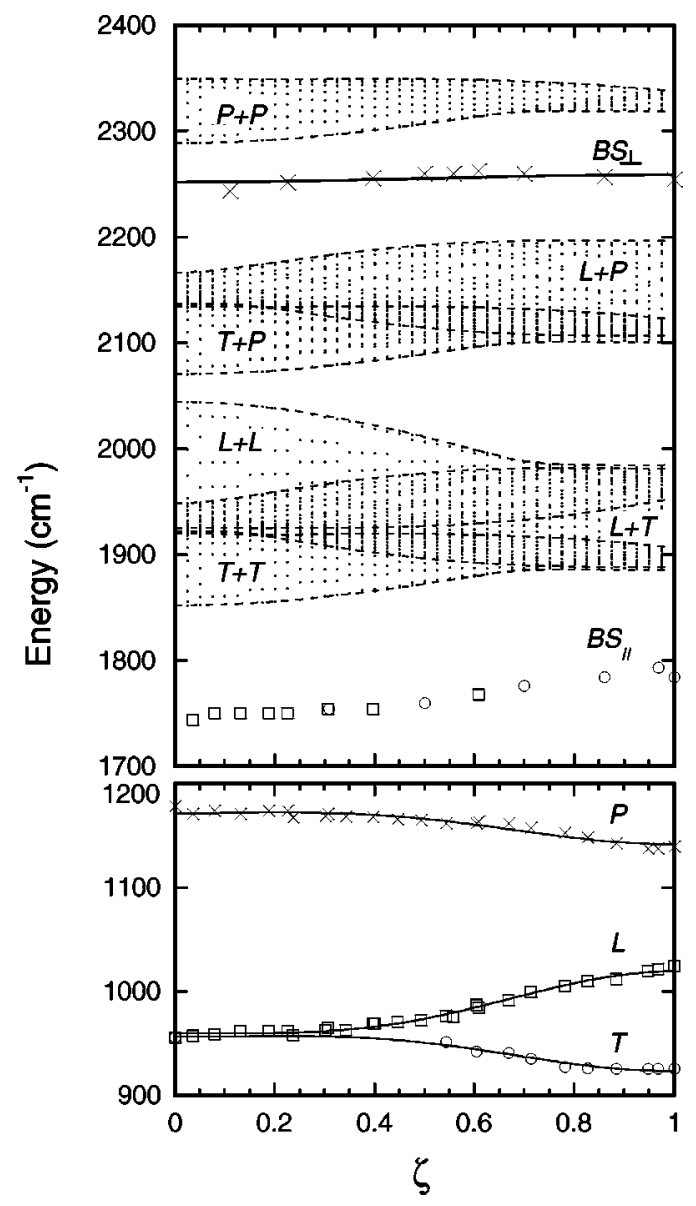

FIG. 3. The dispersions of the one-phonon (lower) and twophonon (upper) states. For the one-phonon states, the crosses represent the perpendicular-polarized mode $(P)$, while squares and circles show the longitudinal $(L)$ and transverse $(T)$ modes, respectively. The corresponding two-phonon bound states are represented by the same symbols. The free two-phonon bands are calculated from the one-phonon dispersion curves. 
which correspond to the double and combination losses such as $L+L$ and $L+P$, respectively. In the calculations we considered one-phonon states only, with the wave vectors parallel to the scattering plane, and neglected the interactions with the overtone states. ${ }^{3}$ The losses due to the free two-phonon states (denoted by $F S$ ) are partly resolved: the losses around 1950 and $2100 \mathrm{~cm}^{-1}$ in Fig. 2(a), where the excitation of the $T$ mode is negligible, are contributed from the $L+L$ and $L$ $+P$ bands, respectively. Note that the adsorption of background CO would cause a separate peak at $1900 \mathrm{~cm}^{-1}$ after prolonged experiments. The calculated density of states (DOS) for these bands at $\zeta=0.11$ is shown in Fig. 1, with the energy resolution $\left(20 \mathrm{~cm}^{-1}\right)$ taken into account. Although the scattering cross sections are not known, the calculated DOS reproduces the observed peak positions very well.

It is apparent from Fig. 3 that the overtone branches lie outside the two-phonon bands, which leads to an interpretation of the overtones as two-phonon bound states. $B S_{\|}$and $B S_{\perp}$ represent the parallel- and perpendicular-polarized bound states, respectively. Here we describe the two-phonon state extremely in a localized basis: (1) the two vibrational quanta are completely localized in a single $\mathrm{H}$ adatom, corresponding to the overtones for isolated species; and (2) the two quanta are distributed in two different $\mathrm{H}$ adatoms. We differentiate the two cases because of the anharmonicity, which lowers the former energy. Upon the lateral coupling being incorporated, the latter forms a free two-phonon band, whereas the former behaves like an impurity state, depending on the anharmonicity. When the anharmonicity is large enough, it is split off from the band, giving rise to a twophonon bound state. The bound state is only weakly coupled to the neighbors or, in other words, to the free two-phonon states, which results in small dispersions compared with the corresponding one-phonon states, as shown in Fig. 3.

In the dispersion curve for $B S_{\|}$(Fig. 3), the squares and circles represent the energies of $B S_{\|}$when the $L$ and $T$ modes dominate in the one-phonon loss region [corresponding to Figs. 2(a) and 2(b)], respectively. Interestingly, both symbols fall in the same curve, which indicates that they are ascribed to an identical bound state. (Note that the corresponding onephonon states are different.) For a localized oscillator of $C_{3 v}$ symmetry, the overtone of the $E$-symmetry fundamental mode exhibits the representation of $A_{1}+E$. The double degeneracy of the $E$ states is lifted when the localization of the bound states is incomplete. Thus the parallel-polarized bound states should exhibit three branches, though we could observe only one. (One of the three is forbidden by the selection rule of impact scattering. ${ }^{1}$ ) These arguments lead us to suggest that the bound state originates from the $A_{1}$ mode, because it appears irrespective of the polarizations ( $L$ or $T$ ) of the one-phonon states. It is not understood why the other branch is not observed.

\section{DISCUSSION}

The anharmonicities and localized properties of the twophonon bound states are discussed with the one-dimensional (1D) model of coupled anharmonic oscillators proposed by Kimball et al. ${ }^{15}$ The model Hamiltonian $H$ for the two- phonon system is represented by

$$
H=\sum_{n} \epsilon a_{n}^{\dagger} a_{n}+\sum_{n, m} \Delta_{n m} a_{n}^{\dagger} a_{m}-\Gamma \sum_{n} a_{n}^{\dagger} a_{n}^{\dagger} a_{n} a_{n},
$$

with the zeroth-order "local" phonon energy $\epsilon$, the "hopping" matrix element $\Delta_{n m}$, and the anharmonicity $\Gamma$ (called $\hbar \omega_{e} \chi_{e}$ in general). $a_{n}^{\dagger}\left(a_{n}\right)$ is the creation (annihilation) operator for the oscillator at the site $n$. For the vibrationally decoupled oscillators $\left(\Delta_{n m}=0\right)$, the eigenvalue for the overtone is deduced to be $2 \epsilon-2 \Gamma$. We can apply this simple model to $B S_{\perp} . B S_{\|}$is the combination of the two orthogonally polarized modes, as noted above; thus the simple 1D model is inadequate for a quantitative analysis.

One-phonon states are described as $E=\epsilon+2 \Delta \cos (\pi \zeta)$, where the $\mathrm{H}-\mathrm{H}$ interaction parameters are neglected except for the nearest neighbors $(\Delta)$. Although this assumption is valid for the bound state due to its localized character, neither of the observed one-phonon branches are well reproduced by this simple function, indicating the coupling to the second-nearest neighbors. ${ }^{14}$ Nevertheless, the zeroth-order energy $\epsilon\left(=x_{0}\right)$ and the interaction energy between the nearest neighbors $\Delta\left(=x_{1} / 2\right)$ are determined to be 1162 and $8 \mathrm{~cm}^{-1}$ by the above fit for the $P$ mode, respectively.

The eigenvalues for the two-phonon bound states were derived from Eq. (1) and are represented by ${ }^{15}$

$$
E_{B}=2 \epsilon-2 \sqrt{\Gamma^{2}+[2 \Delta \cos (\pi \zeta / 2)]^{2}} .
$$

The dispersion width of the bound state is $2 \sqrt{\Gamma^{2}+4 \Delta^{2}}$ $-2 \Gamma$. In this model, the bound state is formed unless $\Gamma$ is zero, and, with increasing $\Gamma$, the bound-state width is reduced and the localization is promoted. We chose $\Gamma$ $=32 \mathrm{~cm}^{-1}$ to reproduce $B S_{\perp}$, and the calculated dispersion curve is shown in Fig. 3 by a solid curve. It should be noted that we consider only one-dimensional coupling in both the experiments and calculations, and the isotope-dilution experiments may be applicable to realize the strictly isolated (two-dimensional decoupled) $\mathrm{H}$ adatoms.

Now that the anharmonicity is derived, we can determine the excitation energies for the decoupled $\mathrm{H}$ adatoms: $v_{0 \rightarrow 1}$ $=1162 \mathrm{~cm}^{-1}$ and $v_{0 \rightarrow 2}=2 v_{0 \rightarrow 1}-2 \Gamma=2260 \mathrm{~cm}^{-1}$. We apply the Birge-Sponer extrapolation ${ }^{1}$ to these values, and roughly estimate the binding energy of $\mathrm{H}$ at the surface along the surface normal direction to be $\sim 1.4 \mathrm{eV}$. The binding energy of $\mathrm{H}$ with respect to the vacuum is $\sim 2.8 \mathrm{eV}$ (Ref. 16) (half of the dissociation energy of $\mathrm{H}_{2}$ plus the adsorption energy), which is much larger than that estimated. On the other hand, the estimated energy is comparable to the barrier of hydrogenation (absorption) with respect to the chemisorption state $[\sim 1 \mathrm{eV}$ (Ref. 17)]. Thus the estimated energy can be attributed to the $\mathrm{H}$ migration barrier to the subsurface octahedral site just beneath the fcc surface site. The barrier energy was theoretically predicted to be $\sim 1 \mathrm{eV} .{ }^{18,19}$ The direct measurement was conducted by Comsa et al., ${ }^{20}$ who determined the barrier with the permeation method to be $\sim 0.3 \mathrm{eV}$ with respect to the vacuum. For comparison, the adsorption energy of $\sim 0.5 \mathrm{eV}$ (Ref. 16) should be added, resulting in a barrier energy of $\sim 0.8 \mathrm{eV}$. Thus, although the 
potential shape of $\mathrm{H}$ at the surface is quite different from that of diatomic molecules, we can obtain insight into the absorption barrier by the overtone analyses.

We can evaluate the localization of the two vibrational quanta from the eigenstates of the bound state deduced from the model calculations. ${ }^{15}$ For the perpendicular-polarized bound state at $\bar{\Gamma}$, the probabilities of the two quanta separated by zero, one, and two sites are 0.94, 0.05, and 0.003, respectively. At a highly excited state of $\mathrm{H}$, the bound state is expected to lie far below the corresponding free multiphonon band, promoting the localization of the vibrational quanta, as observed for the $\mathrm{C}-\mathrm{H}$ vibrational system in molecular benzene. $^{21,22}$ Such a localized property of quanta, in particular for the perpendicular-polarized vibration, is closely related to the absorption ${ }^{9}$ and desorption ${ }^{2}$ dynamics of $\mathrm{H}$ at surfaces from the viewpoint of energy dissipation.

\section{SUMMARY}

In summary, we have measured the multiple losses and overtones of $\mathrm{H} / \mathrm{Ni}(111)$ along the $\bar{\Gamma} \bar{M}$ direction of the surface Brillouin zone, and found that two branches exist outside the free two-phonon bands. This is direct evidence of the existence of two-phonon bound states. Applying the onedimensional model of coupled, anharmonic oscillators, the localized properties of the two-phonon bound state were evaluated.

\section{ACKNOWLEDGMENTS}

This work was supported in part by a Grant-in-Aid from the Ministry of Education, Science, Sports and Culture (Japan).
*Corresponding author telephone: +81-75-753-3979, fax: +81-75753-4000, e-mail: nishijima@kuchem.kyoto-u.ac.jp

${ }^{1}$ H. Ibach and D. L. Mills, Electron Energy Loss Spectroscopy and Surface Vibrations (Academic, New York, 1982).

${ }^{2}$ B. N. J. Persson and Ph. Avouris, Surf. Sci. 390, 45 (1997).

${ }^{3}$ L. J. Richter, T. A. Germer, J. P. Sethna, and W. Ho, Phys. Rev. B 38, 10403 (1988).

${ }^{4}$ P. Guyot-Sionnest, Phys. Rev. Lett. 67, 2323 (1991).

${ }^{5}$ P. Jacob, Phys. Rev. Lett. 77, 4229 (1996); P. Jacob and B. N. J. Persson, J. Chem. Phys. 109, 8641 (1998).

${ }^{6}$ H. Okuyama, T. Ueda, T. Aruga, and M. Nishijima, preceding paper, Phys. Rev. B 63, 233403 (2001).

${ }^{7}$ K. Christmann, R. J. Behm, G. Ertl, M. A. Van Hove, and W. H. Weinberg, J. Chem. Phys. 70, 4168 (1979).

${ }^{8}$ W. Ho, N. J. DiNardo, and E. W. Plummer, J. Vac. Sci. Technol. 17, 134 (1980).

${ }^{9}$ A. D. Johnson, K. J. Maynard, S. P. Daley, Q. Y. Yang, and S. T. Ceyer, Phys. Rev. Lett. 67, 927 (1991).

${ }^{10}$ H. Yanagita, J. Sakai, T. Aruga, N. Takagi, and M. Nishijima, Phys. Rev. B 56, 14952 (1997).
${ }^{11}$ K. Mortensen, F. Besenbacher, I. Stensgaard, and W. R. Wampler, Surf. Sci. 205, 433 (1988).

${ }^{12}$ C. Nyberg and C. G. Tengst $\varnothing$ l, Phys. Rev. Lett. 50, 1680 (1983).

${ }^{13}$ P. Nordlander and S. Holmström, Surf. Sci. 159, 443 (1985).

${ }^{14}$ W. Brenig, Surf. Sci. 291, 207 (1993).

${ }^{15}$ J. C. Kimball, C. Y. Fong, and Y. R. Shen, Phys. Rev. B 23, 4946 (1981).

${ }^{16}$ K. Christmann, O. Schober, G. Ertl, M. A. Van Hove, and W. H. Weinberg, J. Chem. Phys. 60, 4528 (1974).

${ }^{17}$ Hydrogen in Metals I and II, edited by G. Alefeld and J. Völkl (Springer, Berlin, 1978).

${ }^{18}$ D. L. Lynch, S. W. Rick, M. A. Gomez, B. W. Spath, J. D. Doll, and L. R. Pratt, J. Chem. Phys. 97, 5177 (1992).

${ }^{19}$ J. T. Kindt and J. C. Tully, J. Chem. Phys. 111, 11060 (1999).

${ }^{20}$ G. Comsa, R. David, and B. J. Schumacher, Surf. Sci. 85, 45 (1979); 95, L95 (1980)

${ }^{21}$ C. K. N. Patel, A. C. Tam, and R. J. Kerl, J. Chem. Phys. 71, 1470 (1979).

${ }^{22}$ R. G. Bray and M. J. Berry, J. Chem. Phys. 71, 4909 (1979). 